\title{
Optimizing the Performance Evaluation of Robotic Arms with the Aid of Particle Swarm Optimization
}

\author{
K Shivaprakash Reddy \\ Department of Mechanical Engineering, \\ The Oxford College of Engineering, \\ Bommanahalli, Hosur Road, \\ Bangalore - 560068, Karnataka, India
}

\section{Dr.B. Durgaprasad}

Department of Mechanical Engineering, JNTU College of Engineering, Jawaharlal Nehru

Technological University, Anantapur - 515002, Andhrapradesh, India

\author{
Dr.PVK Perumal \\ Department of Aeronautical Engineering, \\ M.A.M School of Engineering, \\ Trichy - Chennai Trunk Road, \\ Siruganur, Trichy - 621105, Tamil Nadu, India
}

\author{
Dr.M.A Murtaza \\ Department of Mechanical Engineering, \\ The Oxford College of Engineering, \\ Bommanahalli, Hosur Road, Bangalore - 560068, \\ Karnataka, India
}

\begin{abstract}
In this modern world, robotic evaluation plays a most important role. In secure distance, this leads the humans to execute insecure task. To acquire an effective result, the system which makes the human task easier should be taken care of and the holdup behind the system should be eradicated. Only static parameters are considered and such parameters are not enough to obtain optimized value in existing work. For consecutively attaining optimized value in our previous work, we focused on both static and dynamic parameters in the robotic arm gearbox model. Now, a genetic algorithm is utilized and the result obtained is greater than the existing work. On the other hand, to attain an effective result the genetic algorithm itself is not enough since it takes massive time for computation process and the result obtained in this computation is not as much closer to the true value. By eliminating all those aforementioned issues, a proper algorithm needs to be utilized in order to achieve an efficient result than the existing and our previous works. In this paper, we anticipated to suggest a Particle Swarm Optimization technique that reduce the computation time as well as make the output result as much closer to the true value (i.e.,) experimentally obtained value.
\end{abstract}

Keywords-Particle Swarm Optimization; Robotic arm gear box; Static\& Dynamic parameters.

\section{INTRODUCTION}

In the physical world, robots are physical agents that attain tasks by manipulation. In general, to sense the environment and effectors to claim physical forces on it robots are equipped with sensors. In the automation process, industrial robots play a most important role in grinding method. The majority of the grinding robots work in a inhibited environment, where instantaneous position and force control is vital [1]. Manipulators, Mobile robots, and Humanoid robots are the three main categories [2]. To enhance product quality and safety, while minimizing costs and processing time, robots find concentrated applications in factories. The robot model depends on the inertia, mass, and center of mass of each link [3]. Tele-manipulators and the capacity of numerical control of machines are the two prominent technologies in which robotics are based on. Tele-manipulators are remotely controlled machines that often hold an arm and a gripper. According to the instructions given by the humans through his/her control device, the movements of arm and gripper will take place. With respect to a given coordinate system, numeric control allows controlling of machines very accurately [2].

\section{A. Types of Robot}

\section{1) Mobile Robots}

A special group of effectors for locomotion, such as wheels, tracks, and legs are used by the mobile robots. The differential drive contains two independently actuated wheels, one on each side. When the movement of both wheels is at equal velocity the robot travels in a straight line. The robot turns on the spot if they move in opposite directions. The development of mobile robots was motivated by the desire to automate transportation in production processes and autonomous transport systems. New types of mobile robots have been created recently like insectoid robots with several legs modeled after examples nature gave us or independent robots for underwater usage.

\section{2) Hard working Robots}

Mostly, in areas of difficult toil robots have been used to replace human workers, which are structured enough for mechanization, like assembly line work in the automobile industry (the classical example) or harvesting machines in the agricultural zone. A few existing examples apart from the assembly robot are Melon harvester robot, Ore transport robot for mines, robot that removes paint from big ships and a robot that creates high precision sewer maps. If robot is used in a proper environment, then it can work faster, cheaper and more exact than human beings.

\section{3) Transporters}

Most autonomous transport are widely in use since the robots still desires environmental changes to find their way. But, designing a robot that can navigate using natural landmarks is probably an end to science fiction. Examples of currently available transporters are (1) Container transporters 
employed to load and unload cargo ships, (2) Medicine and food transport systems in hospices, and (3) Autonomous helicopters employed to transport goods to distant areas.

\section{4) Insensible Steel Giants}

Since robots can be easily protected against hazardous environments and are adequately replaceable, they are used in perilous, toxic or nuclear environments. In some places, for cleaning up a mess robots have been used. For example, in Chernobyl disaster, to clean up the nuclear waste, and also robots are employed to clean grenades and mines all around the world robots have helped. Moreover, robots are sent to Mars and into the depth of the oceans. They can also investigate deep-set ships and can walk on the craters of active volcanoes.

\section{5) Servants and Toys}

In our world, robots may not yet be a common sight, but in several places we already meet and used them. A lot of modern toys like the Sony Aibo are spoiling the today's children's life. To help the older people robots are generally developed to have a better and more secure life. Today, in the name of toys or household helpers they begin to come with us [2].

\section{6) Industrial Robots}

Industrial robots have been entrenched in the manufacturing area, used for performing the tasks such as stacking, casting, painting, sorting, welding, component soldering and more for more than thirty years. This use framework highlights the core value proposal of an industrial robot: performing tasks incessantly and precisely in work environments and scales difficult for humans. For performing operations swiftly, continually and precisely, industrial robots are developed. In the manufacturing industry, operating in relatively static environments and in large numbers, they have a long legacy. To enhance the security and efficiency as well as to decrease the environmental impact, the oil and gas industry suggest the use of industrial robotics. New developments in regions that are difficult or unsafe for humans to work in could be easily handled and maintained by remotely-controlled industrial robots [4].

\section{B. Gear-box}

To change the speed from the low rotating rotor to the high rotating generator a gearbox is used [5]. In their transmissions robotic systems that need to present high torques at the end effectors typically contain high reduction gears, causing some gear-specific friction components to appear, such as position dependent friction. It produces a periodic waveform friction with the frequency by which the two teeth match, since this force happens once a pair of teeth comes together. Thus, as position dependent friction, it has been always considered. The parameters utilize for considering the functioning of gearbox are (1) Direction of turning, (2) Relative speed, (3) The number of revolution, (4) Mechanical advantages concerning the principles of power and speed [6].

Friction takes place along the off line-of-action direction, which lies orthogonal to the line of action, when the power is transmitted along the line of action direction. The major reason for this friction is that the teeth slide together as an alternative of rolling absolutely. Hence, in gearboxes meshing friction is a source of uselessness. The power and load in gear trains are transmitted along the line of action. For small shaft displacements along the line-of-action direction, the relative reduced rigidity of shaft support ball bearings may be responsible, which would guide to torque oscillations and this is called position dependent friction. Though, the meshing friction force in gear teeth is transmitted in the off line-ofaction direction. The friction coefficient between the gear teeth significantly depends on lubricant properties, and it decreases as the relative sliding velocity between gear teeth increases.

\section{Types of Gears}

The amplitude of the oscillation caused by meshing friction also depends greatly on the gear type. Between teeth the working principle of spur or helical gears is rolling. Therefore, in some cases the meshing friction could be small. However, the working principle of other gear types like worm gears, is approximately pure sliding friction. The lubricant film is insufficient to prevent contact between asperities, and friction becomes inflated, when the speed is low. The lubricant film will become adequate to reduce friction, only if speed increases [7].

\section{Common Gearbox Parameters}

Some of the common gear-box parameters are

\section{1) Viscous friction coefficient}

Viscous friction between two surfaces that have relative motion between them relies on dimensional parameters such as contact area and approval between the two surfaces, and also relies on fluid properties, such as fluid specific gravity and viscosity. Between the two meeting surfaces, viscous friction is inversely proportional to the clearance [8].

\section{2) Coulomb friction coefficient}

In contact with each other, coulomb friction is a basic measurement of the friction force that exists between two dry surfaces. The coulomb friction coefficient is a static force, which is to some extent higher than motive force when two materials are at rest whereas in contact with each other. For several simple, pure materials and is given as a unit-less number, this coefficient of friction is distinguished. The coefficient of friction for wood against concrete is 0.62 , for polystyrene against steel is 0.3 to 0.35 , and for steel against Teflon is 0.04 for dry surfaces. To compute the force required to conquer static friction, called as the friction force these numbers are utilized, by multiplying the coefficient of friction times the normal force. The normal force is the mass of the materials times' gravitational pull, with vector calculations added in if the two surfaces are moving up or down an incline against gravitation pull, or towards it [9].

\section{3) Striebeck friction coefficient}

As a function of a dimensionless lubrication parameter $\eta \mathrm{N} / \mathrm{P}$, Striebeck systematically studies the variation of friction between two liquid lubricated surfaces, where $\eta$ denotes the dynamic viscosity, $\mathrm{N}$ represents the speed i.e., revolutions per minute of a bearing, and $\mathrm{P}$ represents the load anticipated on to the geometrical surface [10].

\section{4) Friction smoothness coefficient}

Friction is the resistance that an object encounters when moving over another (OED). Since the sandpaper exerts more 
frictional resistance, it is fast and effortless to drag an object over glass than sandpaper. It was implicit that a surface does not use any frictional force if it is "smooth", in many situations. However, this wouldn't be the case in real life. A "rough" surface is one that provides some frictional resistance [11].

\section{5) Total moment of inertia}

In traditional mechanics, moment of inertia, also called mass moment of inertia, rotational inertia, polar moment of inertia of mass, or the angular mass, (SI units $\mathrm{kg} \bullet \mathrm{m}^{2}$ ) is a measure of an object's resistance to any change in its state of rotation. It is the inertia of a rotating body corresponding to its rotation. The moment of inertia plays much the same role in rotational dynamics as mass does in linear dynamics, depicting the relationship between angular momentum and angular velocity, torque and angular acceleration, and numerous other quantities. The symbol 'I' and sometimes 'J' are often used to represent the moment of inertia or polar moment of inertia [12].

In this paper, by considering the parameter values our primary intention is to decrease the variance occurring between the theoretical value and the practically obtained experimented value. We obtain the optimized parameter value, by utilizing genetic algorithm, which in case positively reduce the error occurred and also the result obtained after applying the optimized parameter value almost bring the intended value and experimentally obtained value.

\section{RELATED WORKS}

The most noticeable features of robotic applications are heterogeneity. With a variety of hardware and software that must be integrated efficiently to develop applications that not only satisfy classic robotic requirements but also software engineering aspects, large robotic projects engage numerous different researchers. However, either they do not cope with such heterogeneity or do not embrace specific robotic requirements in most prior solutions to this problem. In 2008, Juan-Antonio Ferna' ndez-Madrigal et al. [13] have proposed a framework for the implementation of heterogeneous robotic software via a software engineering technique. The BABEL development system, the main phases of the application lifecycle such as, design, implementation, testing, and maintenance are intended to cover when unavoidable heterogeneity conditions are present. For designing and implementing different real robotic applications that employs various programming languages $(\mathrm{C}, \mathrm{C}++, \mathrm{JAVA})$, execution platforms (RT-operating systems, MS-Windows, no operating system at all), communication middleware (CORBA, TCP/IP, USB), and also various hardware components (PC, microcontrollers, and a wide variety of sensor and actuator devices in mobile robots and manipulator arms), the potency of proposed system have been revealed by its support.

In 2008, Sungho Jo [14] has proposed a biologically inspired robotic model. This model is developed combining modified feedback error learning, an unsupervised learning, and the viscoelastic actuator system. They are integrated in order to drive adaptive arm motions, and also discussed the potential efficacy of a biomimetic design of robot skill. With the cerebellar adaptation, the unsupervised learning, the synergy network adaptation, and the viscoelastic system of the muscles the feedback error learning was reliable. To control the redundant actuators efficiently, the proposed model has used a feed forward adaptive approach in the low dimensional control space and an adaptive synergy distribution. With six muscular actuators in the gravitational field, the amalgamation of the two adaptive control approaches has been tested by controlling a two-link planar robot arm. To make smooth, human-like motions, the simulation-based study has shown that the control method can adapt the robot arm motions swiftly and robustly.

Over their rigid counterparts, flexible robot manipulators have abundant advantages. They have increased payload-to weight ratio, they operate at higher speeds, employ less energy and smaller actuators, and they are secure during interaction with their environments. Conversely, light design along with external effects result in components which can oscillate with extreme amplitudes. These oscillations cause deviation from the desired path and long idle periods between tasks in order to perform the intended operation securely and precisely. In 2008, Abdullah Ozer et al. [15] have examined the efficiency of a vibration control method for a two-link flexible robotic arm. Variable stiffness control (VSC) method has been employed to control the excessive oscillations. Due to its dissipative nature, the method was stable and it was relatively insensitive to significant parameter changes and suitable to be implemented on existing robots. Their research considers that the source of the flexibility was either the joints or the links or both. Simulation results have been presented to exhibit the flexibility of the proposed control method. Experiments have been conducted on a laboratory prototype and the results have been presented to prove the validity of simulations.

A human performs a variety of adroit movements by adjusting the dynamic characteristics of his/her musculoskeletal system according to a task involved. By mechanical impedance parameters such characteristics of human movements can be represented. There is a chance that human skillful strategies can be included into robot motion control, if the regulation mechanism of human impedance properties during the task can be clarified and modeled. In 2008, Toshio Tsuji et al. [16] have studied the human hand impedance in preparation for task operations, the so-called "task-readiness impedance", in a virtual ball-catching task. For contact tasks by computer simulations using measured task-readiness impedance they have also discussed a bio-mimetic impedance control of robotic manipulators.

In 2009, Freeman et al. [17] have developed an experimental test facility. This facility was developed for the use by stroke patients in order to enhance the sensory-motor function of their upper limb. Subjects have been seated at the workstation and their task is to continually follow reaching trajectories that are projected onto a target above their arm. To perform this, they used a voluntary control with the addition of electrical stimulation mediated by advanced control methods applied to muscles in their impaired shoulder and arm. The particulars regarding the design of workstation and its periphery systems have been given, together with a depiction of its use during the healing of stroke patients.

In 2009, Yunquan Sun et al. [18] have performed robotic belt grinding operations. This was done by increasing a work piece to the end effecter and imposing it to move along a route 
while maintaining contact with the belt grinding wheel. To give a smooth finish on the work piece, a constant contact force throughout the grinding process was essential, but it was tricky to maintain this force due to a multitude of installation, manipulation, and calibration errors. The proposed methodology for robotic belt grinding has been described, which mainly concentrates on system calibration and force control to enhance grinding performance. For each step of the proposed method have been shown, the overall theory has been explained and experimental results of turbine blade grinding.

Antagonistic Driven Compliant Joints (ADCJs) are object, drawn substantial attention in current robotics research, representing one of the most extensively applied solutions. This was developed to develop human-like and safe joints for human-robot communication. In 2010, Nicola Vitiello et al. [19] have proposed a sensor less torque control technique, appropriate for ADCJs actuated robots. Off-line characterization of the flexibility of the actuation units, defined by the force-elongation curve and online estimation of the force exerted by each actuation unit, through a direct measure of the joint angle, and of the "resting position" of each actuation unit are the two steps followed in the proposed technique. To develop two autonomous force controllers, the proposed force estimation technique has been employed, with no need of additional torque sensors that can be then fused to control the resulting joint torque. Over the shoulder and the elbow ADCJs of the 2-link 2-DOFs planar robotic arm NEURARM, the performance of the proposed torque control has been analyzed. The technique was proved to work effectively, achieving better performances on the test platform, and represents a suitable alternative to modern sensor-based torque controls.

In 2011, Celso De La Cruz et al. [20] have developed a dynamic model of a robotic wheel chair. This was developed considering a lateral deviation of the center of mass. To design a tracking and positioning adaptive control for the robotic wheel chair, the Lyapunov and input/output stability theories have been utilized. Regarding to its matrices and parameters, properties of the dynamic model have been exhibited. A filter has been engaged to obtain a closed loop equation that permits designing of adaptive control law. Consequently, to improve the adaptive control in the sense of eliminating parameter drift a projection algorithm has been used. Experimental results have shown the better performance of the adaptive control.

In 2011, C.M. Wronka et al. [21] have proposed a dynamic model of a robotic manipulator mounted on a moving base. This was designed by means of the Euler-Lagrange technique. In the dynamic equations, it is assumed that the base inertia was large enough not to be affected by the manipulator motion and hence can be treated as a time-varying parameter. To a Mitsubishi PA10-6CE robotic manipulator mounted on a 2DOF platform, the presented derivation has been applied. By comparing simple closed-loop control results of the simulated model with experimental data from the manipulator mounted on the platform the model has been evaluated.

In real environments, achieving manipulation tasks interactively necessitates a high level of precision and stability. One must provide the robot with the skill to react quickly to abrupt changes in the environment at the same time when one cannot assume a fully deterministic and static environment. In 2012, Ashwini Shukla et al. [22] have recorded the kinematics of arm and fingers of human subjects. This was done during unperturbed and perturbed reach and grasp motions. After the onset of the motion the target's location has been changed abruptly in the perturbed demonstrations. Between the hand transport and finger motions, data has shown a strong combination. To seamlessly and rapidly adapt the finger motion in coordination with the hand posture, they theorize that the coupling enables the subject. A coupled dynamical system based controller has been proposed, whereby two dynamical systems driving the hand and finger motions have been coupled to provide their robot with the capability. For reach-to-grasp motions the proposed method has provided a compact encoding that ensures fast adaptation with zero latency for re-planning. They have proved that this coupling ensures smooth and "human-like" motions from the simulation performed on the real iCub robot.

In 2012, Hassan Azarkish et al.[23] have presented the performance of the particle swarm optimization and the genetic algorithm compared as a typical geometry design problem. From a given fin volume, the plan maximizes the heat transfer rate. The analysis presumes that a linear temperature sharing the length of the fin. Using the B-spline curves the fin profile generated and restricted by the alteration of control point coordinates. An inverse method applied to find the appropriate fin geometry yield the linear temperature distribution along the fin corresponds to optimum design. The numbers of the populations, the count of iterations and time to convergence measure efficiency. For geometry optimization, results show that the particle swarm optimization is most competent.

\section{PARTICLE SWARM OPTIMIZATION BASED OPTIMIZED PARAMETER VALUE}

\section{A. Generation of Chromosomes}

Initially, numbers of chromosomes are generated randomly. Each chromosome has number of genes. The randomly generated chromosomes can be determined as,

$$
C_{i}^{(k)}=\left\{c_{0}^{(k)}, c_{1}^{(k)}, c_{2}^{(k)}, \cdots, c_{N-1}^{(k)}\right\}
$$

Here, $C_{i}$ - No of Chromosomes, $C_{j}$ - No of genes in Chromosomes, and every ithvalue has its own range, which should be different from the range of other genes, and the minimized range will give better result when compared to the existing work.

Fitness computation

$E_{e r r}=(E v-M v)$
$S_{e l}=\sum_{x=1}^{N_{x}} E^{x} e r r$
$m=\frac{S_{e l}}{N_{x}}$
If $\min (\mathrm{m})$
End If




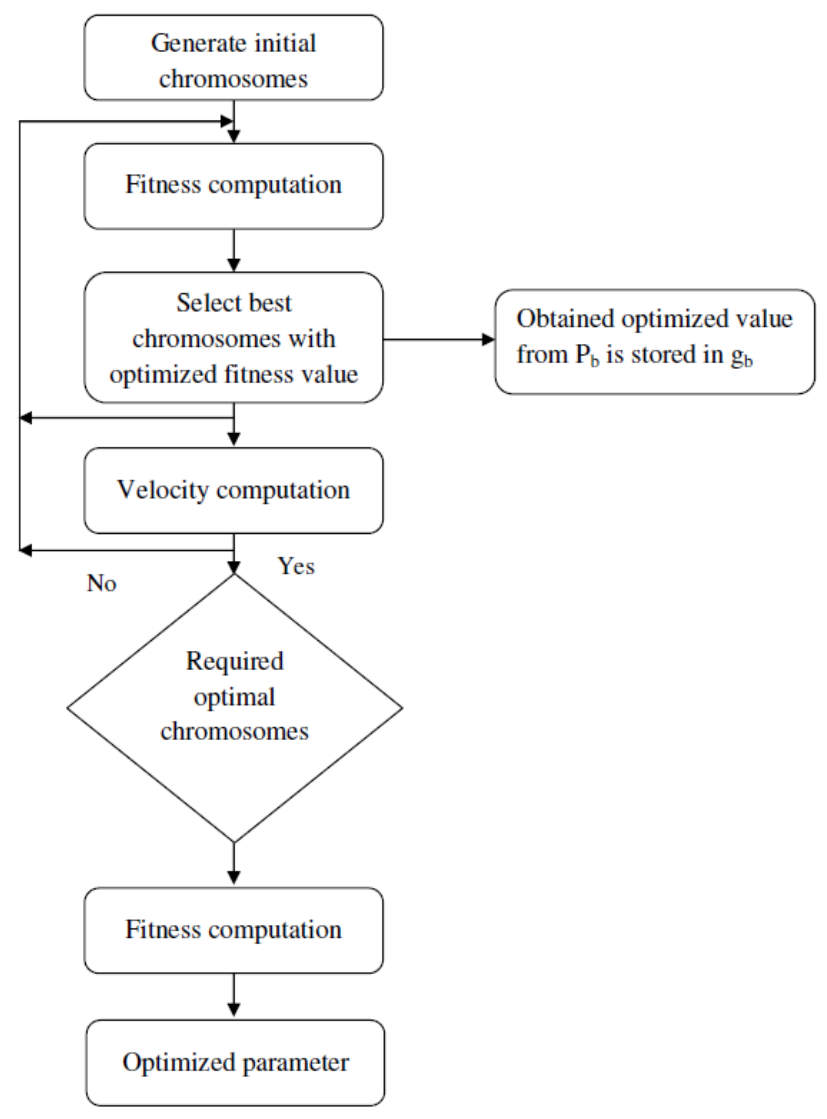

To compute the fitness, all the obtained chromosomes and the corresponding parameter values are taken from the eqn (1). Here, error minimization is the fitness function, which is shown below.

Where, Ev - Experimental value; Mv - model value ; Error Element ; - sum of error Elements ; - No of Elements; - Mean of error elements, which is also said to be Fitness. From the above pseudo code, select number of chromosomes to be applied with the genetic operations for velocity computation.

Objective Function

$$
\dot{x}(t)=\left[\begin{array}{c}
x_{3}-x_{4} \\
x_{4}-x_{5} \\
\frac{1}{I_{m}}\left(-T_{s t}\left(x_{1}\right)-D_{g}\left(x_{3}-x_{4}\right)-T_{f r}\left(x_{3}\right)+u\right) \\
\frac{1}{I_{g}}\left(T_{s t}\left(x_{1}\right)+D_{g}\left(x_{3}-x_{4}\right)-k_{s} x_{2}-D_{a}\left(x_{4}-x_{5}\right)\right) \\
\frac{1}{I_{a}}\left(k_{s} x_{2}+D_{a}\left(x_{4}-x_{5}\right)\right) \\
I=I_{a i}+I_{m i}+I_{g i}
\end{array}\right]
$$

$I_{m i}=$ Moment of inertia of motor

$I_{a i}=$ Moment of inertia of arm

$I_{g i}=$ Moment of inertia of gear box

$T_{\text {stq }}=$ Spring Torque
$D_{p a}=$ Damping Parameters of arm

$D_{p g}=$ Damping parameters of gearbox

$k_{S S}=$ Stiffness of the second spring

$T_{f r}\left(x_{3}\right)=C_{v} x_{3}+\left(C_{c}+C_{c s} \operatorname{sech}\left(\sigma x_{3}\right)\right) \tanh \left(\tau x_{3}\right)$

$=\mathrm{Viscous}$ friction coefficient

$=$ Coulomb friction coefficient

$=$ Striebeck friction coefficient.

$=$ Friction torque

$$
T_{s t t}\left(x_{1}\right)=k_{g b 1} x_{1}+k_{g b 3} x_{1}^{3}
$$

$=$ Gearbox stiffness parameter 1

$=$ Gearbox stiffness parameter 2

$=$ Spring Torque

$$
E_{r}(\theta)=\frac{1}{N} \sum_{t=1}^{N} e^{2}(t, \theta)
$$

Is said to be the Error value

$$
e(t, \theta)=y(t)-y(\hat{t}, \theta)
$$

If , then the simulated output of the model is obtained with the input $\mathrm{u}(\mathrm{t})$ and without $\mathrm{e}(\mathrm{t})$ for the current parameter vector. The criterion (6) is minimized by an iterative numerical search algorithm, which involves simulation of the system for different values of . [24]

$$
\min _{\theta} E_{r}(\theta)=\frac{1}{N} \sum_{t=1}^{N} e^{2}(t, \theta)
$$

\section{B. Velocity computation}

Particle Swarm Optimization has two significant operators: velocity update and position update. Particle Swarm Optimization utilizes numerous particles to investigate minimum values of an objective function.

Every particles move in a certain search space with a velocity. Based on the current velocity of each iteration and, a newly obtained particle are calculated. The velocity and position of the particle will be updated according to the equations given below.

$$
\begin{aligned}
& \quad v_{m}=v_{c}+l_{f 1} r_{1}\left(p_{b}-p\right)+l_{f 2} r_{2}\left(g_{b}-p\right) \\
& p_{m}=p+v_{m} \\
& v_{c}=\text { Current velocity vector } \\
& p=\text { Current position vector } \\
& p_{m}=\text { Modified position vector } \\
& r_{1}=\text { Random parameter } 1(0-1) \\
& r_{2}=\text { Random parameter } 2(0-1) \\
& l_{f 1}=\text { Learning Factor } 1
\end{aligned}
$$


$l_{f 2}=$ Learning Factor 2

$p_{b}=$ Previous best $\left(p_{\text {best }}\right)$

$g_{b}=$ Best value tracked by PSO $\left(g_{\text {best }}\right)$

\section{Selection of best chromosomes}

After the completion of number of iterations, the best chromosome is chosen from the obtained chromosomes. Here, the best chromosome is one having least error value. Subsequently, the genes of best chromosome are arranged in the rising order and the chromosome that has least error is preferred as the best gene.

From the above process, we have obtained the optimal fitness value i.e., eqn (8). By utilizing this fitness value, we can obtain the value similar to that of the experimental value [24].

\section{RESUlt AND DisCUSSION}

To obtain the optimized fitness value we are utilizing particle swarm optimization which is more preferable for optimization process here we use optimize fitness value for four sets of experiment (i.e.) Estimation, Validation1, Validation2, Validation 3.which gives more accurate value when compare it with the standard value as well as the value which obtain from our previous technique genetic algorithm.

In table I shows the optimized fitness value in the proposed column which clearly shows that the proposed optimized fitness value is greater than that of existing fitness value and our previous work, the corresponding graph show below figure. 1 clearly explains that our proposed work is more closely near to the experimental value. The standard and existing data are obtained from [25].In the figure 2,3,4,5 explains the general model graph for standard and the existing work amid blue color marked is how much occur in the black color shaded graph. The remaining portion shows that Existing work is lag with the standard value.

TABLE I. COMPARISON OF EXISTING FITNESS VALUE, FIRST WORK FITNESS VALUE AND PROPOSED FITNESS VALUE

\begin{tabular}{|c|c|c|c|}
\hline Experiments & $\begin{array}{c}\text { Existing } \\
\text { Fitness Value }\end{array}$ & $\begin{array}{c}1^{\text {st }} \text { work } \\
\text { Fitness Value }\end{array}$ & $\begin{array}{c}\text { Proposed } \\
\text { Fitness Value }\end{array}$ \\
\hline Estimation & 72.63 & 78.7 & 85.14 \\
\hline Validation 1 & 73.22 & 79.2 & 85.56 \\
\hline Validation 2 & 8.887 & 37.5 & 63.6 \\
\hline Validation 3 & 95.86 & 98 & 98.36 \\
\hline
\end{tabular}

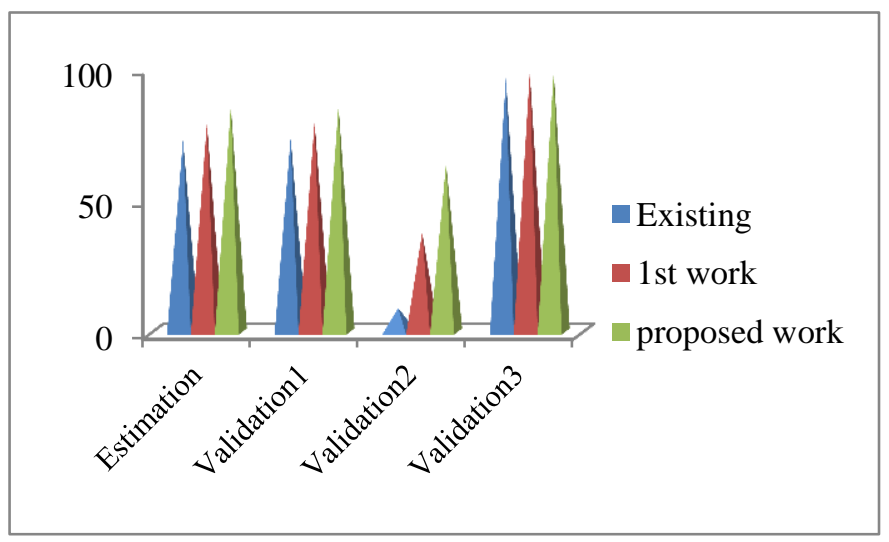

Figure 1. (Fitness comparison)
Graph obtained for Standard and Existing Experimental value for four sets of Experiment

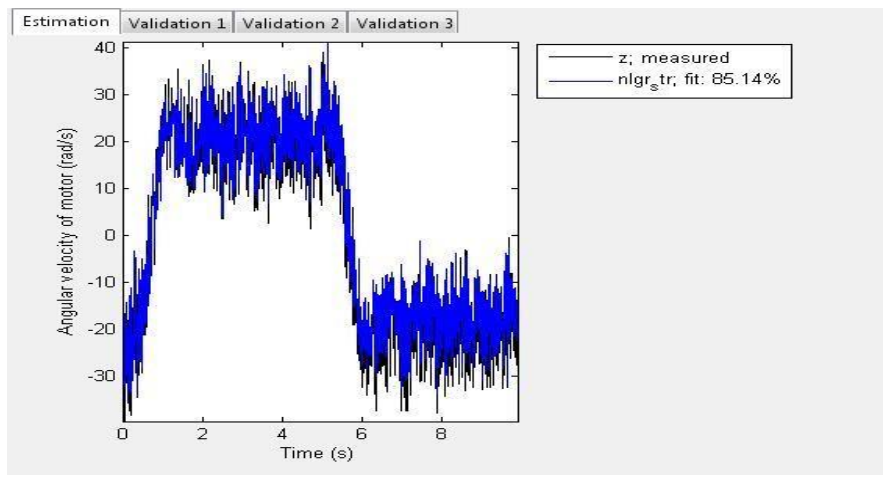

Figure 2. (Estimation)

In Existing work they attain $72.63 \%$ of fitness value when compare to that of standard value. (i.e.) the blue color occur only $72.63 \%$ in the black shaded part in the graph. In our previous work we attain $78.7 \%$. In this proposed work we attain $85.14 \%$ which is greater than that of Existing work and also our previous work.

In Existing work they attain $73.22 \%$ of fitness value when compare to that of standard value. (i.e.) the blue color occur only $73.22 \%$ in the black shaded part in the graph. In our previous work we attain $79.2 \%$. In this proposed work we attain $85.56 \%$ which is greater than that of Existing work and also our previous work.

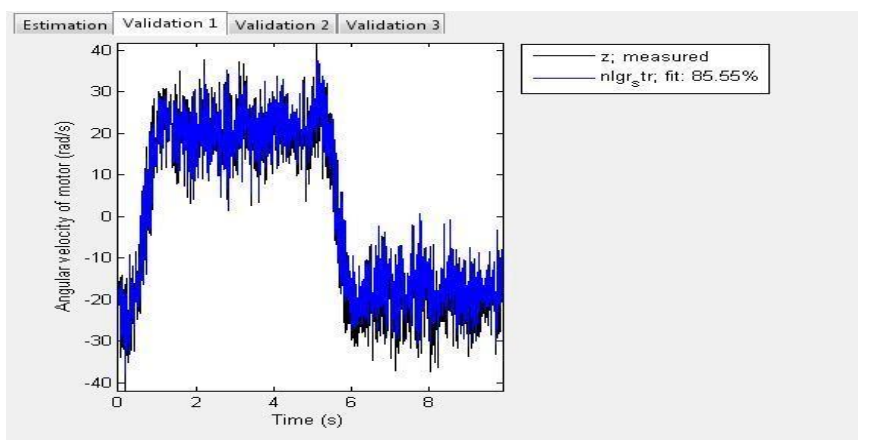

Figure 3. (Validation 1)

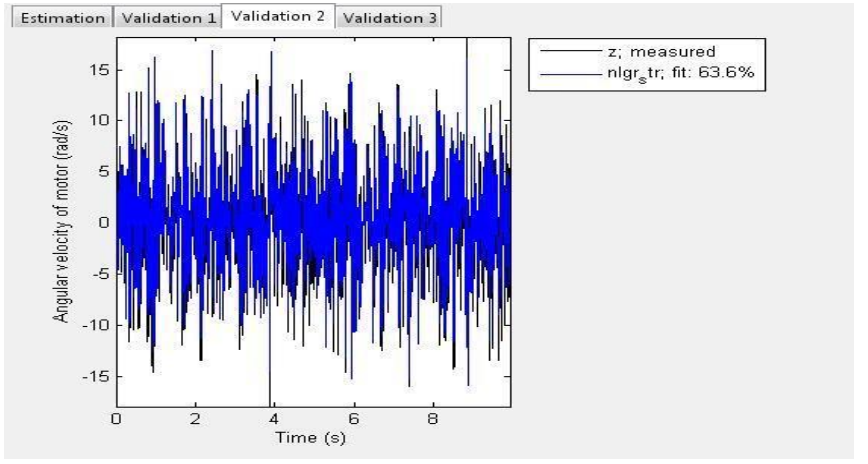

Figure 4. (Validation 2)

In Existing work they attain $8.887 \%$ of fitness value when compare to that of standard value. (i.e.) the blue color occur only $8.887 \%$ in the black shaded part in the graph. In our 
previous work we attain $37.5 \%$. In this proposed work we attain $63.6 \%$ which is greater than that of Existing work and also our previous work.

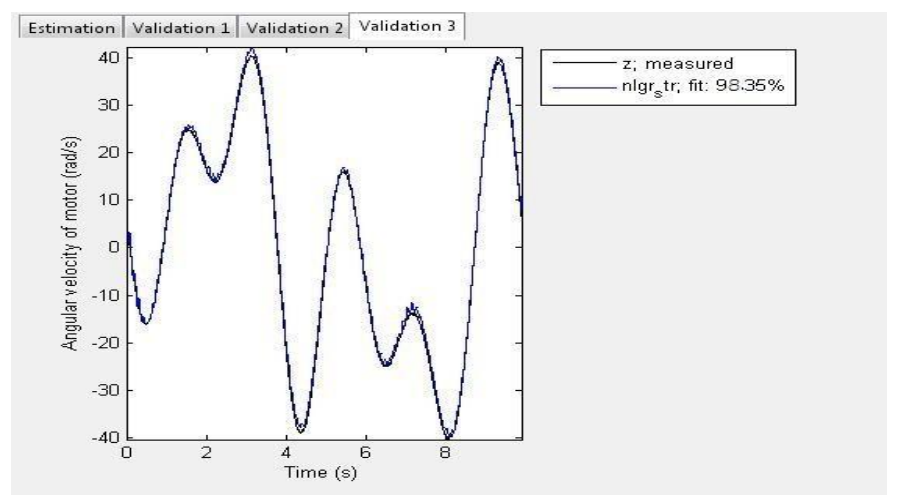

Figure 5. (Validation 3)

In Existing work they attain $95.86 \%$ of fitness value when compare to that of standard value. (i.e.) the blue color occur only $95.86 \%$ in the black shaded part in the graph. In our previous work we attain $98 \%$. In this proposed work we attain 98.36\% which is greater than that of Existing work and also our previous work.

\section{CONCLUSION}

The proposed technique was robust and is used to predict the optimized value from the generate chromosomes. In general there is some conflict in experimentally obtained value and numerically obtained value. In order to eradicate those conflicts we have to optimize the parameter value and minimize the error occur in those equation, which certainly gives the optimized value which is closely nearer to that of experimentally obtained value. The obtained optimized value should certainly improve the flexibility of robotic arm.

\section{REFERENCE}

[1] Saeid Nahavandi, Mohammad Jashim Uddin, Yasuo Nasu, Hieu Trinh, Mozafar Saadat, "Automated robotic grinding by low-powered manipulator" ,Robotics and Computer-Integrated Manufacturing, Vol.23, pp.589-598,2007

[2] Tim Niemueller and Sumedha Widyadharma," Artificial Intelligence An Introduction to Robotics", AI-Robotics, July 8, 2003

[3] Basilio Bona and Aldo Curatella," Identification of Industrial Robot Parameters for Advanced Model-Based Controllers Design", Proceedings of the 2005 IEEE International Conference on, Robotics and Automation (ICRA), pp 1681 - 1686, 2005.

[4] Clint Heyer, "Human-Robot Interaction and Future Industrial Robotics Applications", IEEE/RSJ International Conference on Intelligent Robots and Systems (IROS), 2010

[5] Svend Gade, Richard Schlombs, Christoph Hundeck and Christian Fenselau," Operational Modal Analysis on a Wind Turbine Gearbox", Conference \& Exposition on Structural Dynamics, 2009

[6] Joan M. Chambers, Mike Carbonaro and Hana Murray," Developing conceptual understanding of mechanical advantage through the use of
Lego robotic technology", Australasian Journal of Educational Technology, v24 n4 p387-401 2008

[7] S.E. Hodges and R.J.Richards, "Look and Learn: Towards Cheap, Flexible Robots", Proceedings of the International Conference on Recent Advances in Mechatronics ICRAM 1995.

[8] Medhat K. Bahr Khalil," Estimated versus calculated Viscous Friction Coefficient in Spool Valve Modeling", Technical Conference on IFPE, 2008.

[9] Coulomb Friction (http://www.wisegeek.com/what-is-coulombfriction.html)

[10] Tribology (http://en.wikipedia.org/wiki/Tribology)

[11] The Coefficient of (http://www.mathsrevision.net/alevel/pages.php?page=79)

Friction

[12] Moment of inertia (http://en.wikipedia.org/wiki/Moment_of_inertia)

[13] Juan-Antonio Fernandez-Madrigal, Cipriano Galindo, Javier Gonza' lez, Elena Cruz-Martin and Ana Cruz-Martın, "A software engineering approach for the development of heterogeneous robotic applications" ,Robotics and Computer-Integrated Manufacturing,Vol.24,pp.150166,2008

[14] Sungho Jo, "Adaptive biomimetic control of robot arm motions", Neurocomputing, Vol.71, pp.3625-3630, 2008

[15] Abdullah Ozer and S. Eren Semercigil, "An event-based vibration control for a two-link flexible robotic arm: Numerical and experimental observations", Journal of Sound and Vibration, Vol.313, pp.375-394, 2008

[16] Toshio Tsuji and Yoshiyuki Tanaka, "Bio-mimetic impedance control of robotic manipulator for dynamic contact tasks", Robotics and Autonomous Systems, Vol.56, pp.306-316, 2008

[17] C.T. Freeman, Hughes, Burridge, Chappell, Lewin and Rogers, "A robotic workstation for stroke rehabilitation of the upper extremity using FES”, Medical Engineering \& Physics, Vol.31, pp.364-373, 2009

[18] Yunquan Sun, DavidJ. Giblin and Kazem Kazerounian, "Accurate robotic belt grinding of work pieces with complex geometries using relative calibration techniques", Robotics and Computer-Integrated Manufacturing, Vol.25, pp.204-210, 2009

[19] Nicola Vitiello , Tommaso Lenzi, Stefano Marco Maria De Rossi, Stefano Roccella and Maria Chiara Carrozza, "A sensor less torque control for Antagonistic Driven ,Compliant Joints", Mechatronics,Vol.20,pp.355-36,2010

[20] Celso De LaCruz, Teodiano Freire Bastos, Ricardo Carelli, "Adaptive motion control law of a robotic wheel chair" ,Control Engineering Practice,Vol.19,pp.113-125,2011

[21] C.M. Wronka and M.W. Dunnigan, "Derivation and analysis of a dynamic model of a robotic manipulator on a moving base", Robotics and Autonomous Systems, Vol.59, pp.758-769, 2011

[22] Ashwini Shukla and Aude Billard, "Coupled dynamical system based arm-hand grasping model for learning fast adaptation strategies", Robotics and Autonomous Systems, Vol.60, pp.424-440, 2012

[23] Hassan Azarkish, Said Farahat, and S.Masoud H. Sarvari, "Comparing the Performance of the Particle Swarm Optimization and the Genetic Algorithm on the Geometry Design of Longitudinal Fin ",World Academy of Science, Engineering and Technology,Vol.61,pp.836839,2012

[24] [24] K Shivaprakash Reddy,PVK Perumal,B. Durgaprasad and M.A Murtaza, "Optimizing the Performance Evaluation of Robotic Arms with the Aid of Genetic Algorithm" , International Journal of Computer Applications, Vol. 51, No. 5, pp.24-30, 2012

[25] Erik Wernholt and Svante Gunnarsson, "Nonlinear Identification of a Physically Parameterized Robot Model", LiTH-ISY-R-2739, pp.1-6, Aug 2006 